\title{
Programa para las Culturas Municipales y Comunitarias. Secretaria de Cultura y el Café Científico La Paz
}

DOI: $10.46932 / \mathrm{sfjdv2n1-070}$

Received in: November 1st, 2020

Accepted in: December 30th, 2020

Biol. Cruz del Carmen Juárez Olvera

Socia Titular Somedicyt

E-mail: cjuarez@ipn.mx

\section{M. en C. Indra María Álvarez Ramírez}

CODICE (*). Profesor/investigador Centro Interdisciplinario de Ciencias Marinas-IPN. E-mail: indra.alvarez@gmail.com

Q.B.P. José Francisco Piña Puente

E-mail: jbotanico@hotmail.com

Esteban Fernando Félix pico

Master science, researcher/professor

Current Institution CICIMAR-IPN

Full address: And. Bahia Magdalena 134, Sudcalifornia FOVISSSTE, La Paz, BCS, 23080 México. E-mail: bajamangle@yahoo.com

\section{RESUMEN}

La Comunidad Sudcaliforniana de Divulgadores de la Ciencia A.C. (CODICE), apoya la Comunicación Pública de la Ciencia en Baja California Sur, que cuenta con instituciones de investigación y un gran número de científicos, cuyas acciones buscan atender la problemática a nivel: estatal, regional y nacional; Por lo que es necesario divulgar el conocimiento científico que se genera en estos centros y "decodificar" la información científica en mensajes amenos y atractivos que vinculen: ciencia, sociedad y cultura, en beneficio de la sociedad. Las actividades de CODICE, han permitido el acercamiento a la ciencia a través de actividades culturales, democratizando el conocimiento científico; de esta forma ha sido posible contar con la participación de diversos sectores de la sociedad, participando niños, jóvenes, adultos, estudiantes, empresarios, amas de casa, personas de la tercera edad pensionados jubilados y discapacitados. El proyecto Café Científico La Paz, se ha desarrollado de manera continua desde 2007, el último martes de cada mes, aún antes de haberse conformado CODICE demostrando su continuidad y permanencia, con el propósito ser un foro informal de discusión que permite a la gente acercarse a pensar y platicar la ciencia, a disfrutarla. Las sesiones se realizan en un restaurant que permite un ambiente agradable, tranquilo en donde los asistentes degustan la bebida o comida de su preferencia, durante el desarrollo de la charla. Los integrantes del Proyecto somos divulgadores de la ciencia, quienes se han profesionalizado en esta tarea de comunicación científica. Se obtuvo apoyo durante 2014-2015; a través de la Dirección de Culturas Populares y el Instituto Sudcaliforniano de Cultura, este último convoca a participar en el apoyo a Culturas Populares y Comunitarias (PACMYC). En este trabajo se presentan resultados obtenidos a través de las diferentes sesiones, los temas presentados en el espacio del Café Científico, así como las sesiones llevadas a cabo extramuros: Telesecundaria del Poblado de San Pedro B.C.S., Centro Municipal de las Artes, Centro de Capacitación Técnico-Industrial, sesión especial para la Asociación de Ciegos y Débiles visuales.

Palabras clave: Educación no formal, divulgación de la ciencia, cultura popular. 


\section{INTRODUCCIÓN}

\subsection{COMUNIDAD SUDCALIFORNIANA DE DIVULGADORES DE LA CIENCIA A.C.”}

Se funda en 2008 como una agrupación conformada de comunicadores de la ciencia, inquietos en compartir el conocimiento científico que se genera, a través de mensajes que resulten amenos, alegres, atractivos e interesantes, que vinculen la ciencia, conocimiento, arte y cultura en beneficio de la sociedad sudcaliforniana.

Cabe destacar que los integrantes de la Comunidad Sudcaliforniana de Divulgadores de la Ciencia, como profesionales en ciencias, se han profesionalizado a través de Talleres y Diplomados en Comunicación Publica de la Ciencia. Bajo esta modalidad, el grupo ha participado en eventos de Ciencia y Tecnología organizados a nivel nacional, estatal e internacional.

\subsection{EL PROYECTO DE CAFÉ CIENTÍFICO DE LA PAZ}

Este proyecto del Café Científico La Paz, es una propuesta de acercamiento de la ciencia a la cultura, a través de charlas que se desarrollan mensualmente, con el propósito de ser espacios de interacción y acercamiento con la sociedad en diferentes áreas de la ciudad. Compartiendo el conocimiento científico y avance tecnológico, para que el ciudadano tenga al alcance la información, se apropie de este conocimiento y lo aplique en mejorar su calidad de vida. Este proyecto inicio en 2007 y se lleva a cabo de manera ininterrumpida el ultimo martes de cada mes, como una iniciativa continua y permanente. Se lleva a cabo el último martes de cada mes. Se abordan temas de las ciencias sociales, ciencias exactas, ciencias naturales, ciencias de la salud. Temáticas diferentes. Asisten niños, jóvenes, adultos, estudiantes, empresarios, amas de casa, personas de la tercera edad pensionados jubilados y discapacitados.

Las sesiones se realizan en un restaurant que permite un ambiente agradable, tranquilo en donde los asistentes degustan la bebida o comida de su preferencia, durante el desarrollo de la charla.

Los objetivos del café científico son los siguientes:

Contribuir a la integración del conocimiento de la ciencia hacia los diferentes sectores de la sociedad.

Integrar a los especialistas y académicos de la localidad con los sectores sociales interesados en sus contribuciones.

Favorecer la cultura científica y contribuir hacia la conformación de una ciudadanía mejor informada.

Promover que la ciencia forme parte de nuestra cultura, en espacios no académicos. 
Favorecer la educación no formal, relacionada con los conocimientos académicos y tecnológicos fuera de las instituciones de investigación en la entidad.

En esta etapa, el proyecto Café Científico La Paz, además de llevarse a cabo de manera mensual, pretende salir a centros de reunión en colonias lejanas al centro de la ciudad, el apoyo que se otorgue al proyecto será de utilidad para llegar a esas comunidades y llegar a más público.

Cabe destacar que las sesiones de Café Científico La Paz, se transmiten en tiempo real a través del internet; los videos de las sesiones quedan disponibles en YouTube, para quien desee acceder a ellas en esta modalidad. Utilizar la tecnología para difundir el Café Científico La Paz, permite que este proyecto se conozca a nivel local, a nivel nacional e internacional. Actualmente este proyecto forma parte de la red internacional de Cafés Científicos, a través del sitio http://www.cafescientifique.org/.

Este trabajo se presentó como ponencia oral durante el XXI Congreso Nacional de Divulgación de la Ciencia y la Técnica (Juárez Olvera et al, 2016). En este Congreso se conmemoró el 30 Aniversario de la formación de la Sociedad Mexicana para la Divulgación de la Ciencia y la Técnica, A.C. (SOMEDICYT A.C.), sitio https://somedicyt.org.mx/ .

\section{METODOLOGÍA.}

Para cada sesión de Café Científico, se diseñó la papelería correspondiente y alusiva al tema. A cada uno de los asistentes se les entregaron señaladores de libros durante las sesiones del café científico del mes de febrero, marzo y abril de 2015. Para darle difusión a cada una de las sesiones se elaboraron carteles y se colocaron en distintas instituciones educativas, culturales y de turismo (Fig. 1).

Figura 1.- Carteles del café científico ¡Va a la calle! del mes de mayo centro municipal de las artes y del mes de junio: la estevia hoja dulce ancestral.

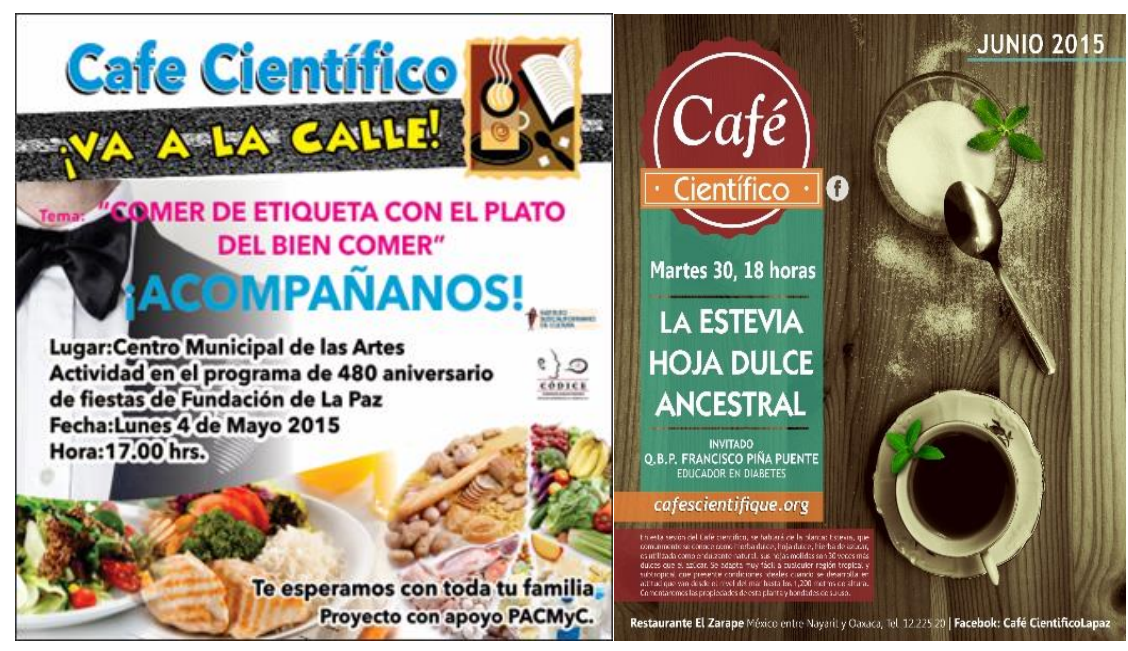


Se contó con apoyo del Departamento de Difusión del Instituto Sudcaliforniano de Cultura, para emisión de boletines de prensa y entrevistas en medios de comunicación, en Radio Cultura www.radioculturaisc.com, quedando grabadas las entrevistas de los ponentes. Café Científico fue invitado el 8 de marzo al programa Frecuencia Juvenil de Radio UABCS, 1190 AM, dando tiempo suficiente para exponer el proyecto, su historia, alcances y metas. Derivado de esta entrevista, se invitó al ponente de la sesión del mes de abril (Fig. 2).

Figura 2.- Entrevistas en Radio y Televisión estatal, en el Programa Recorrido Cultural. Difusión www.radioculturaisc.com. En Radio UABCS, Programa Frecuencia Juvenil, 1190 AM, programación en vivo.

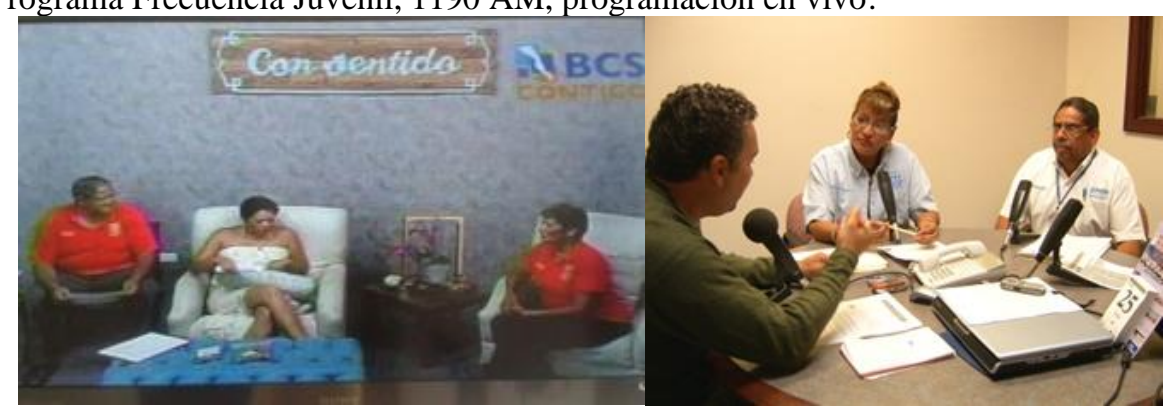

Se continúo con la difusión a través de las redes sociales, prensa local y agenda cultural del Instituto Sudcaliforniano de Cultura (Fig. 3).

Figura 3.- Notas publicadas en el periódico local el Sudcaliforniano de la editora nacional OEM.

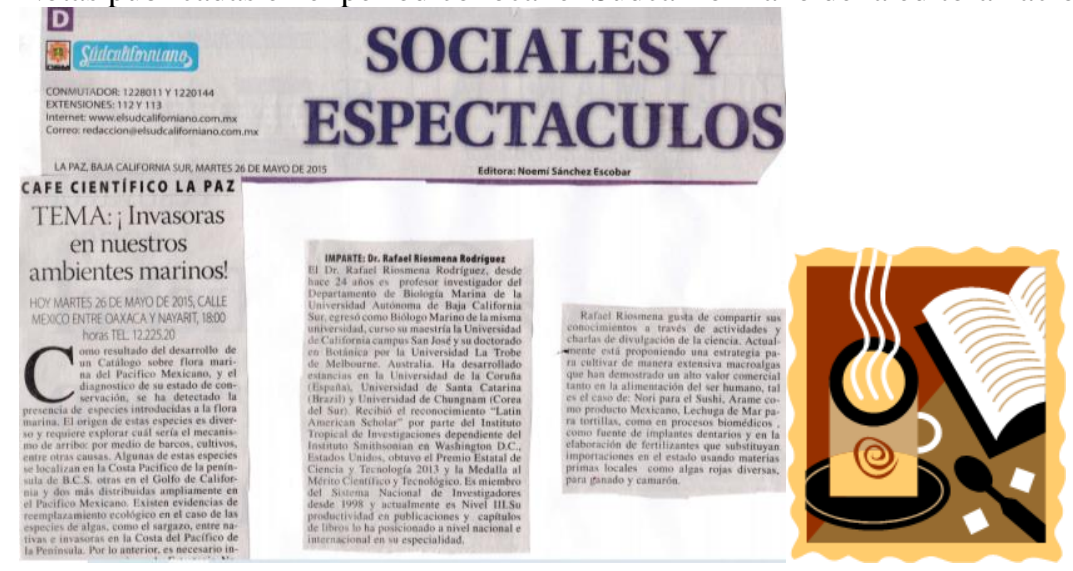

Durante las sesiones la recepción de los asistentes es apoyada por estudiantes de posgrado voluntarios, para registro de asistencia y dinámica de presentación y cierre del Café Científico con una libreta de comentarios (Fig. 3). 
Figura 3.- Las sesiones son apoyadas por estudiantes del CICIMAR-IPN, con la transmisión en vivo vía Facebook, con la toma del registro de asistentes, libreta de comentarios y materiales de la presentación.

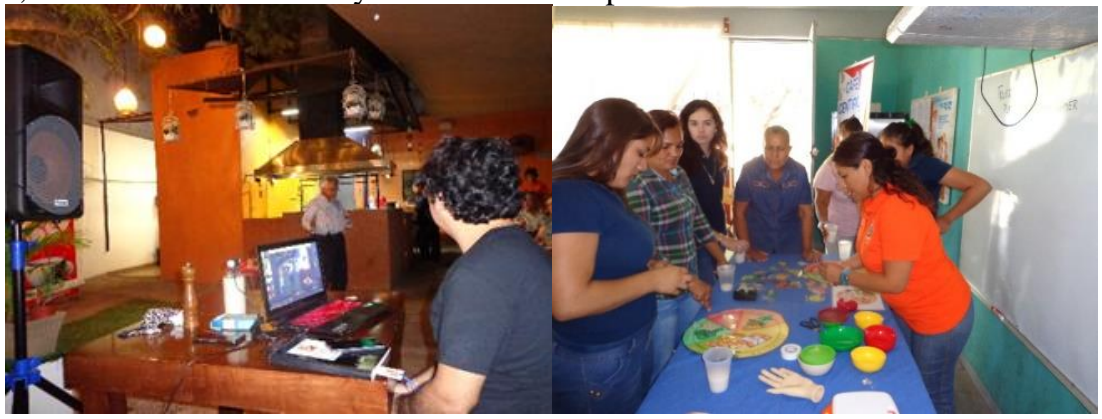

Los asistentes a las sesiones del Café Científico participaron a través de preguntas, comentarios y opiniones relativas al tema.

\section{RESULTADOS}

Cada año iniciamos el último martes de enero con una sesión celebrando el aniversario de inicio del Café Científico (Fig. 4). En esta sesión se recopilan las sesiones del año anterior, invitando a los ponentes a que comenten su experiencia; a cada uno de ellos se les da como obsequio un recuerdo y se les brinda café con pastel; asimismo se invita a los ponentes del año que inicia, para que nos den una semblanza y comentarios de su tema a exponer.

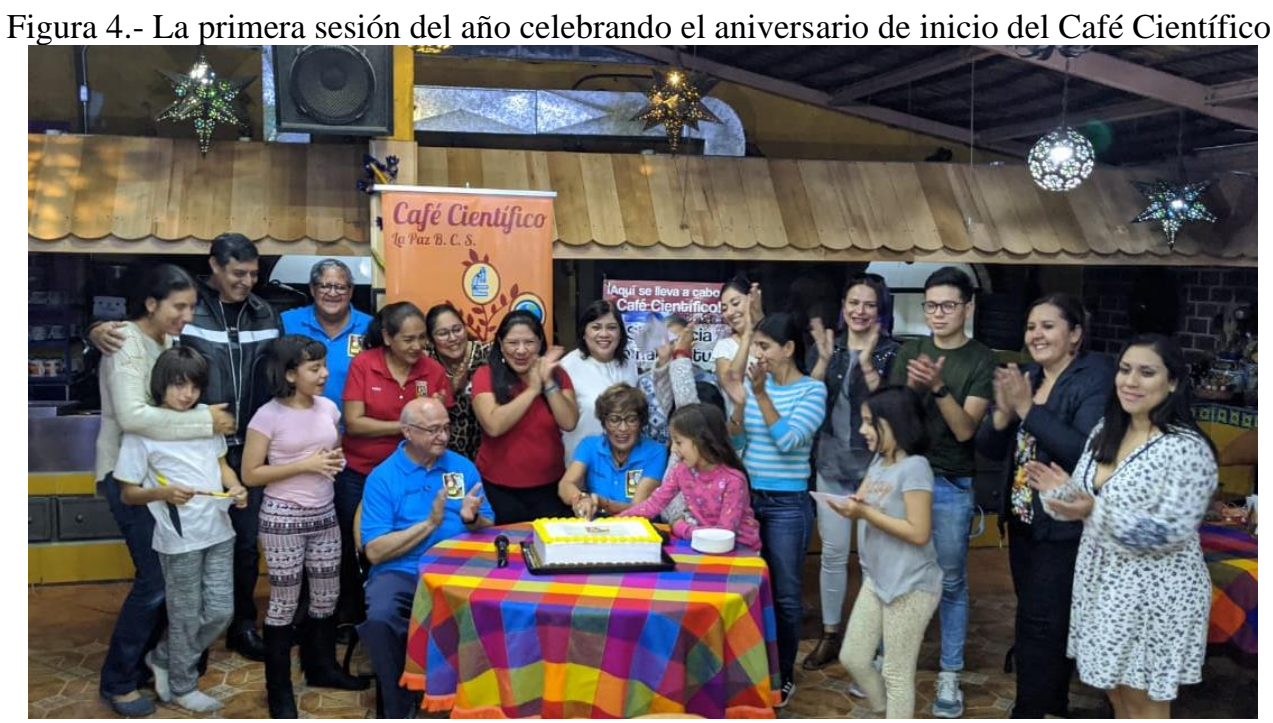

Durante la sesión del Café Científico de febrero de 2015 titulado "Lo que me cuentan tus bigotes", como ponente la M. en C. Sofia López Alvirde, después de la exposición del tema la participación de los asistentes se dio de manera espontánea, al contar con material que favoreció la interacción, por ejemplo, colmillos y cráneos de lobos marinos (Fig. 5). 
Figura 5.- La sesión de febrero con la charla "Lo que me cuentan tus bigotes", como ponente la M. en C. Sofia López Alvirde, con dibujos de lobos marinos coloreando por los niños.

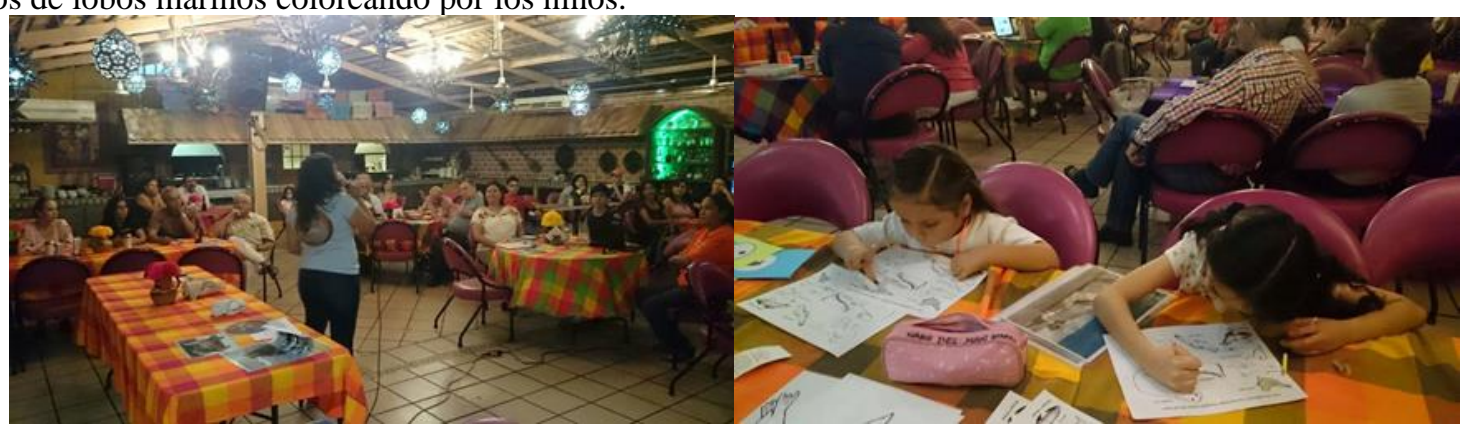

En la sesión de marzo 2015 se tituló “¡Algas Hasta En La Sopa!” como ponente Alejandra Mazariegos y Karla León Cisneros, en este caso la interacción de los asistentes después de escuchar la conferencia, se les presentó material bibliográfico como las guías para identificación, así como algas secas y productos de uso común que contienen algas, los niños participaron coloreando dibujos de macroalgas. Y los asistentes degustaron platillos orientales preparados con macroalgas (Fig. 6).

Figura 6.- La sesión de “¡Algas Hasta En La Sopa!” tuvo el objetivo dar a conocer los usos alimenticios que tienen las macroalgas marinas.

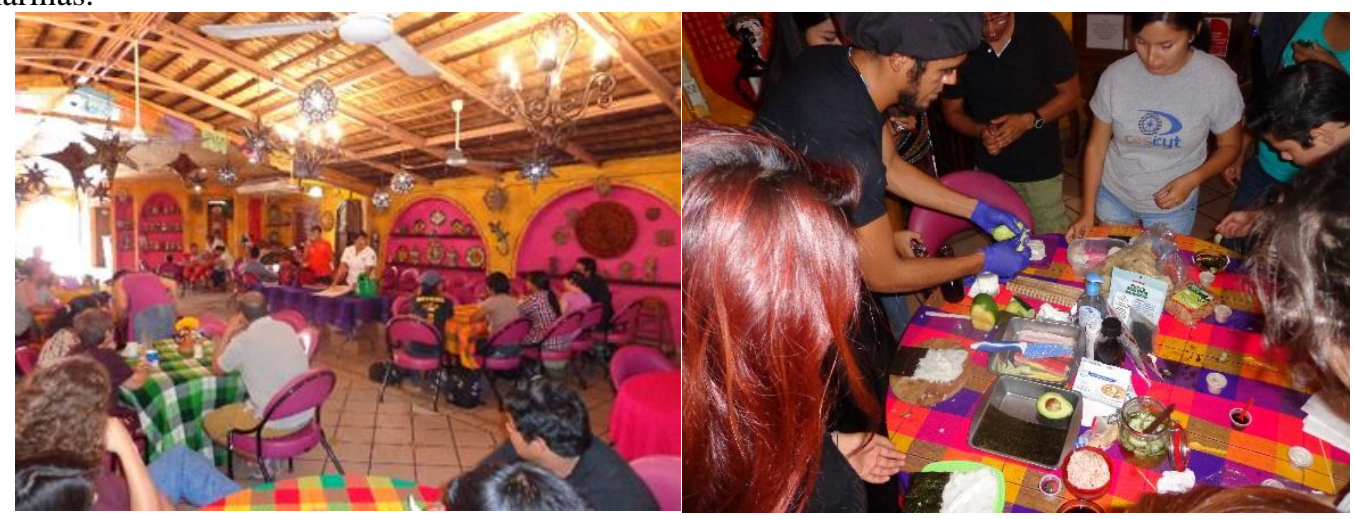

La sesión extra del Café científico se dio el 17 de abril de 2015, fue por la invitación al telebachillerato de San Pedro, B.C.S. (Situado a $25 \mathrm{~km}$ de la ciudad de La Paz), posicionó al proyecto fuera del sitio donde regularmente se desarrolla, de ahí que esta modalidad se le denominó "¡Café científico sale a la calle!", lo que favoreció una mayor participación de la comunidad que recibe el café científico (Fig. 7). El tema de la conferencia trató sobre la alimentación familiar y sus nutrimentos con el título "Comer de etiqueta con el plato del bien comer", fueron los ponentes: IBQ. Lucía Soto Simental, M. en C. Sofía López Alvirde, Biol. Cruz del Carmen Juárez y Q.B.P. Francisco Piña Puente. A los asistentes de la comunicad se les solicito trajeran productos de su despensa para leer las etiquetas y comparar las diferentes presentaciones. 


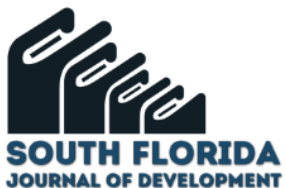

Figura 7.- Fuera de la habitual sede de la ciudad de La Paz, la modalidad se le denominó “¡Café científico sale a la calle!” con la conferencia sobre la alimentación familiar y sus nutrimentos.

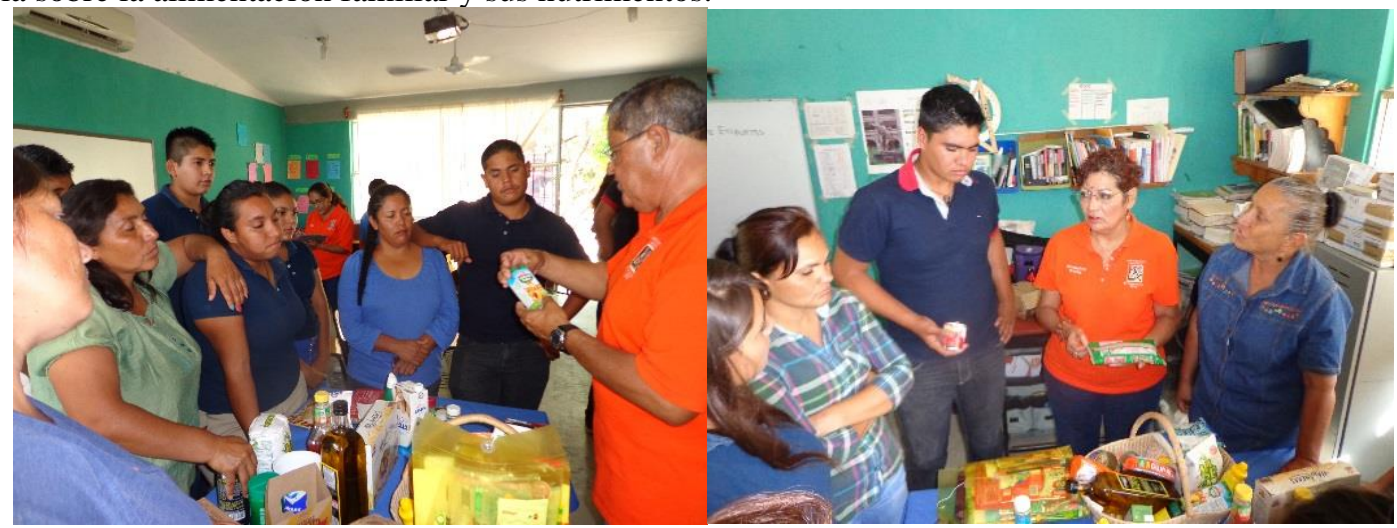

Se llevó a cabo la sesión del Café Científico de abril de 2015, con el tema titulado “¿Vida En lo más profundo del Océano? Fauna Abisal”, del expositor Harif Salas, cabe destacar que es un joven divulgador de la ciencia de tan solo 12 años y entonces estudiante de secundaria (Fig. 8).

Figura 8.-Nuestro divulgador más joven que nos habló del mundo marino, apoyado con pinturas de especies de invertebrados y mamíferos marinos, que el mismo realizó con su arte.

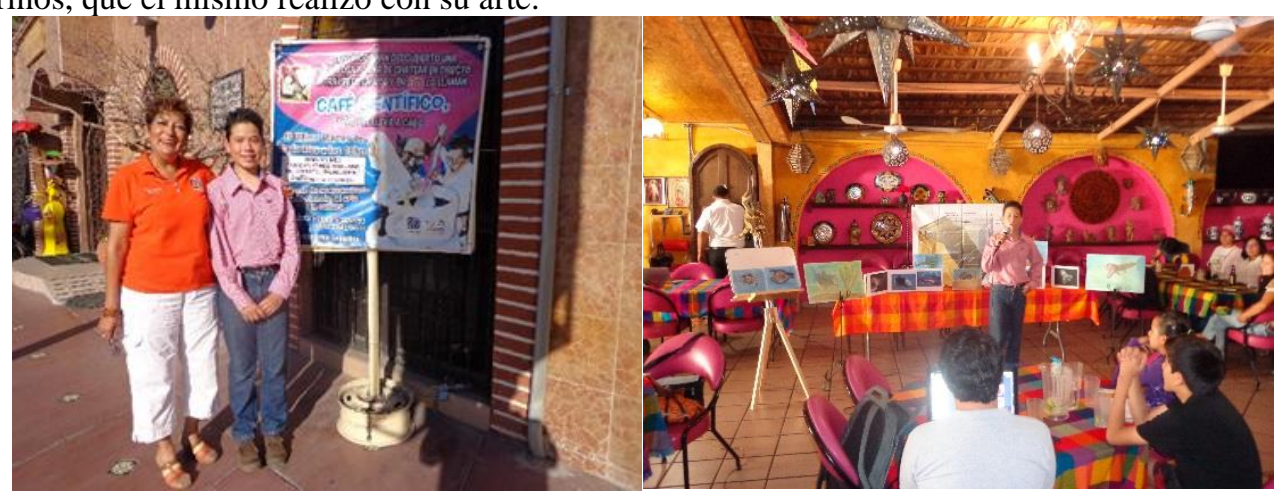

Otra sesión extra del Café científico se dio el 3 de mayo 2015, con la modalidad ¡Sale a la Calle! Por invitación del Centro Municipal De Las Artes durante las fiestas de celebración de la Fundación De La Paz (Fig. 9). Repitiendo con el tema “El Plato del Bien Comer y Lectura De Etiquetas”. De la misma forma fuimos apoyados la IBQ. Lucia Soto Simental, M. en C. Sofia López Alvirde, Biol. Cruz del Carmen Juárez y Q.B.P. Francisco Piña Puente. 
Figura 9.- Durante las fiestas de celebración de la Fundación De La Paz, fuimos invitados para presentar el tema "El Plato del Bien Comer y Lectura De Etiquetas".

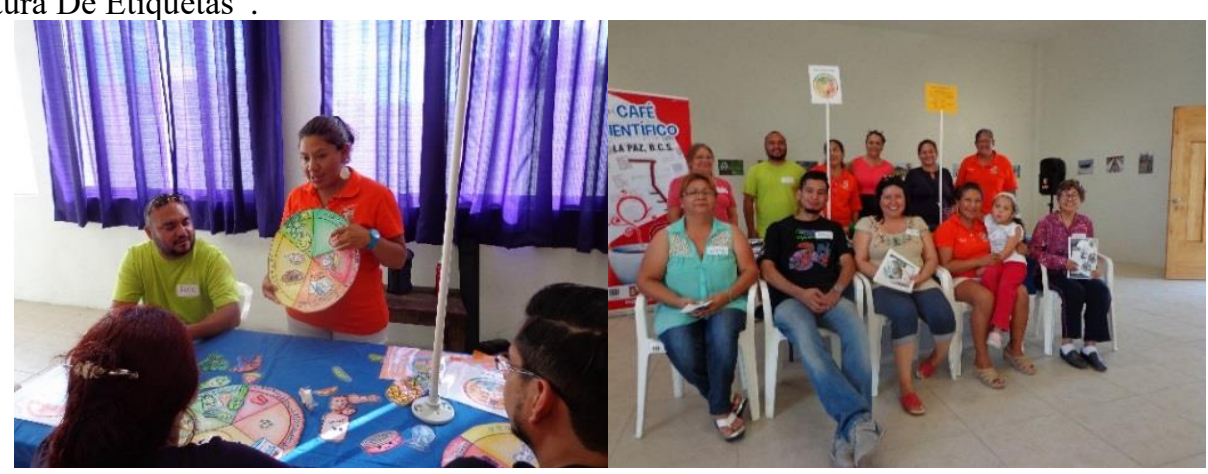

La siguiente sesión correspondió al Café Científico de mayo de 2015, con el tema “¡Invasoras en Nuestros Ambientes Marinos!” un tema ambiental que hace referencia a las especies introducidas o exóticas, por el Dr. Rafael Riosmena Profesor/Investigador de la Universidad Autónoma de Baja California Sur. En esta sesión los asistentes tuvieron oportunidad de observar material conservado en el herbario de la institución (Fig. 10).

Figura 10.- El Dr. Rafael Riosmena con el tema ambiental “¡Invasoras en Nuestros Ambientes Marinos!” sobre la problemática que causan algunas macroalgas en el golfo de California y costas del Pacífico.

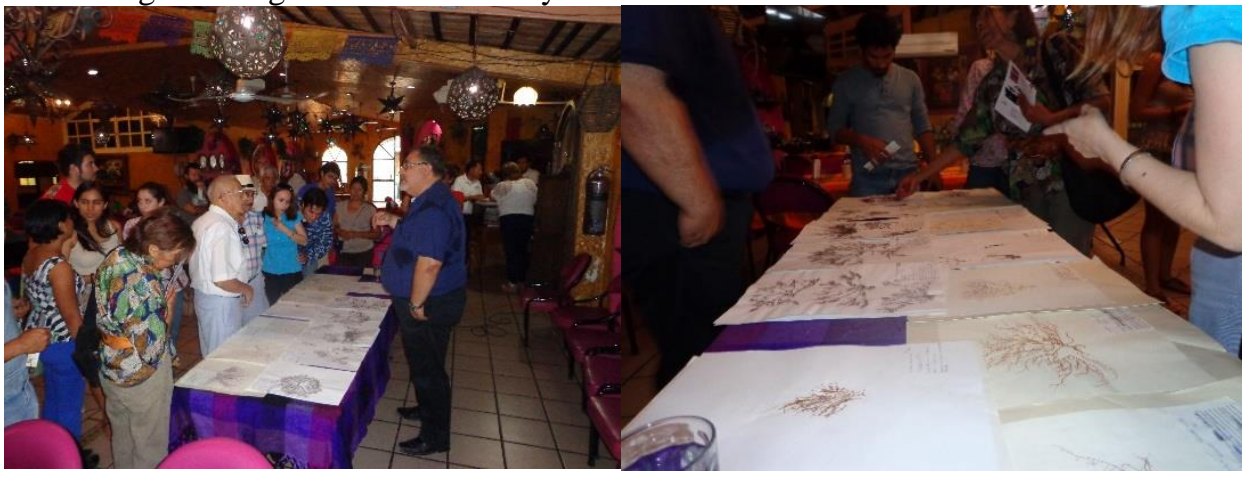

Para terminar con las sesiones del primer semestre correspondió al Café Científico de junio 2015, con la disertación de la conferencia "La Estevia: Hoja Dulce Ancestral” por el Q.B.P. José Francisco Piña Puente, impartió interesante charla referente a la nutrición funcional para pacientes con Diabetes, él representa al Club Dulce Recuperación que son pacientes con Diabetes del Hospital general "Dr. Carlos Estrada Ruibal" ISSSTE, La Paz BCS (Fig. 11). 


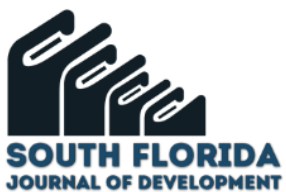

Figura 11.- Una charla referente a la nutrición funcional para pacientes con Diabetes, recomendando el consumo del edulcorante la hoja de estevia.

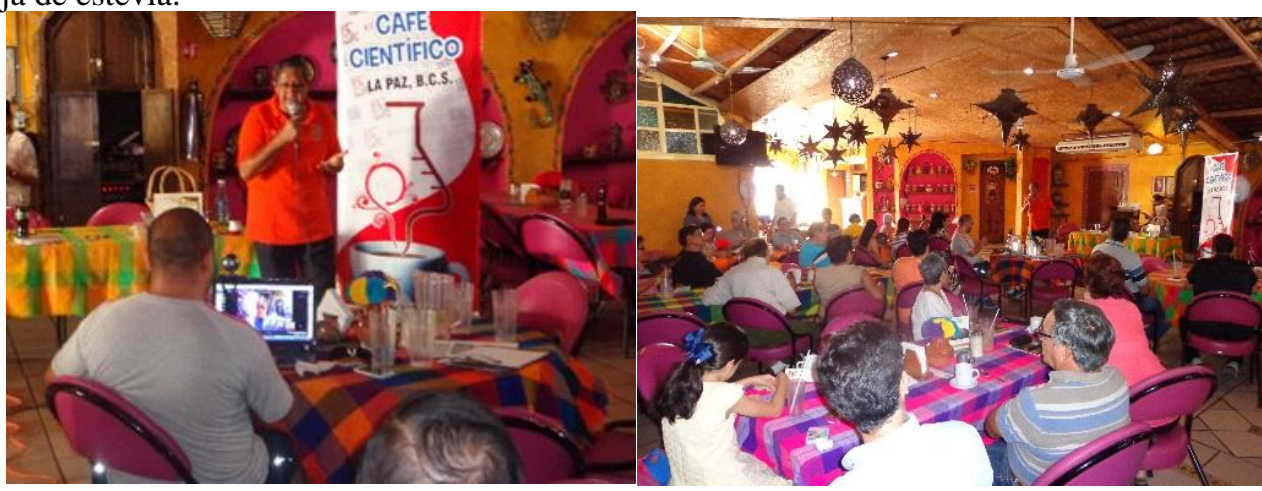

El segundo semestre iniciamos el Café Científico en agosto de 2015 con una interesante charla titulada "En tu Sangre...la solución a varios problemas" por la Dra. Norma Álvarez González especialista en medicina del deporte (Fig. 12). En esta charla se reprodujo la técnica para extracción del plasma de la sangre de un voluntario, y se le implantó inyectado a un paciente con problemas de desgaste en las articulaciones, como fue el caso de rodilla.

Figura 12.- la Dra. Norma Álvarez González especialista en medicina del deporte nos demostró como es la técnica que utiliza en su clínica para rehabilitar las articulaciones dañadas por desgaste.

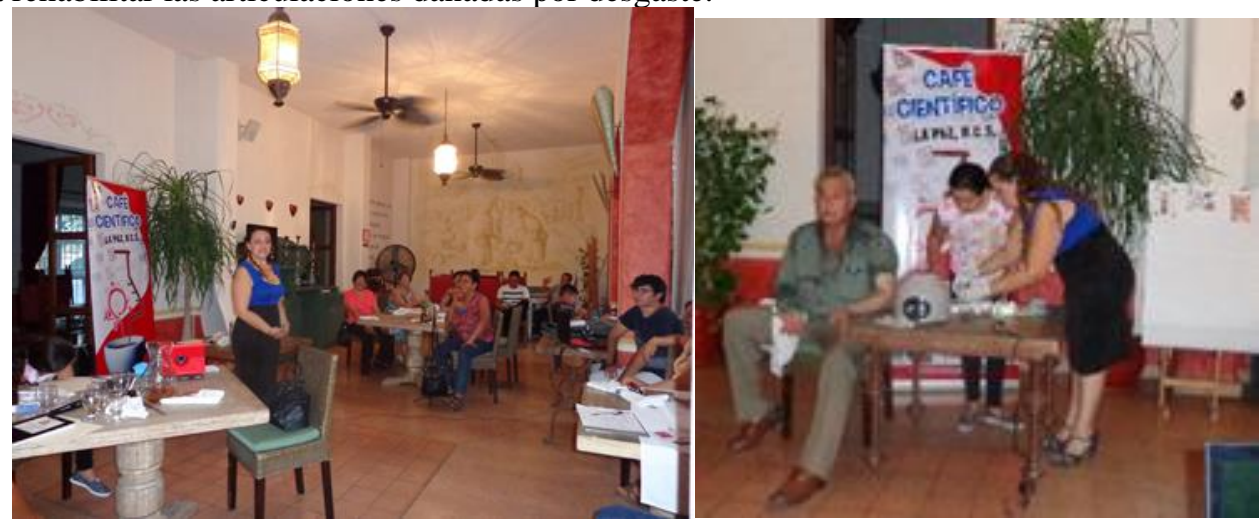

Para el Café Científico de septiembre de 2015 el tema fue "Ballenas y Delfines de sudcalifornia, su importancia económica y ecológica" por el Dr. Jorge Urban Profesor/Investigador de la Universidad Autónoma de Baja California Sur (Fig. 13). En los mares de la península de BC se desarrolla una importante actividad turística que consiste en el avistamiento de ballenas y delfines de distintas especies que arriban a las costas, especialmente durante la temporada de invierno. Se tuvo un acercamiento a barbas de ballenas, colmillos y otras piezas dentales, así como una guía de identificación de estos organismos. 


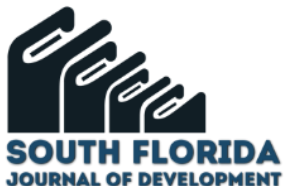

Figura 13.- El tema de las ballenas es muy atractivo y el Dr. Jorge Urban con su experiencia nos dio una explicación muy rica en conocimientos de la biología y comportamiento de los cetáceos.

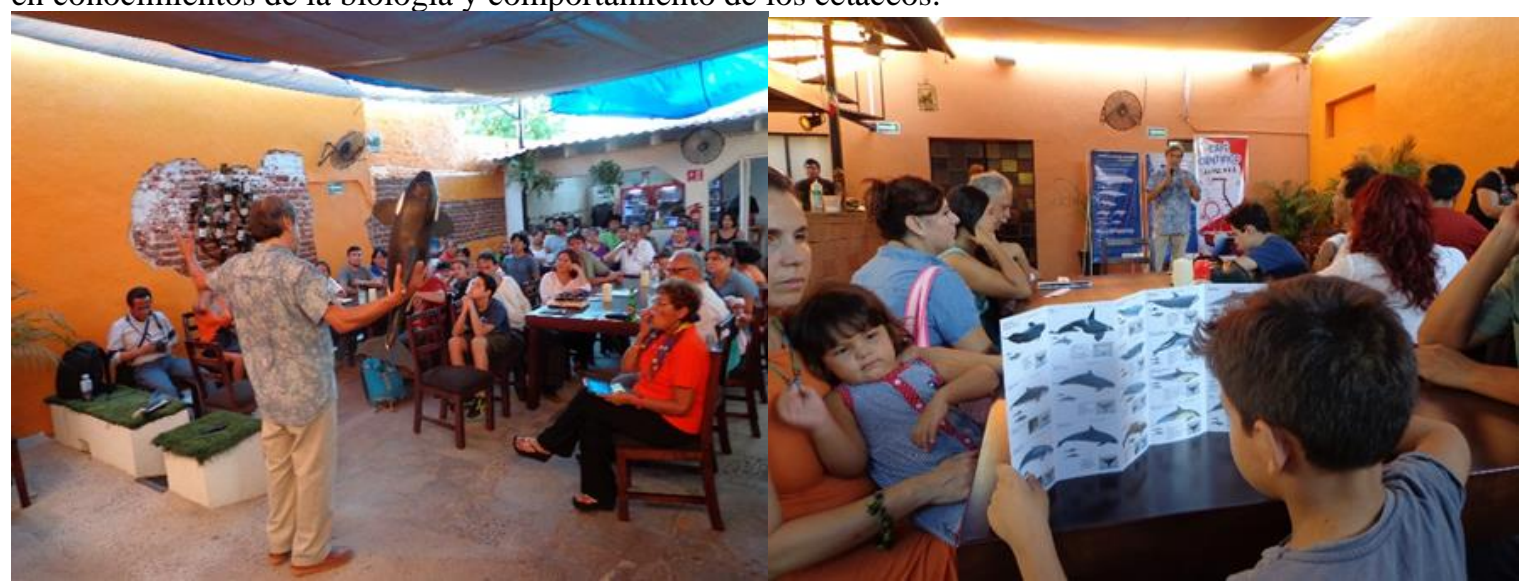

Una tercera sesión extra del Café Científico en octubre de 2015, con la modalidad ¡Café Científico Sale a la Calle! Con el tema "El Plato del Bien Comer". Se llevó a cabo en la sala de usos múltiples del CECATI, invitados por la Asociación de Ciegos y Débiles visuales, A.C. Por los ponentes el IBQ. Lucía Soto Simental, Biol. Cruz del Carmen Juárez y Q.B.P. Francisco Piña Puente (Fig. 14). Previamente se solicitó a los participantes que aportaran diversos alimentos, frutas y verduras, en esta sesión permitió que cada uno de los invidentes propusiera los elementos de un desayuno, comida y cena, organizando en grupo ellos solos el platillo de su elección. Fue una experiencia significativa para el equipo.

Figura 14.- Con el tema "El Plato del Bien Comer" esta se llevó a cabo en la sala de usos múltiples del CECATI, con invitados por la Asociación de Ciegos y Débiles visuales.

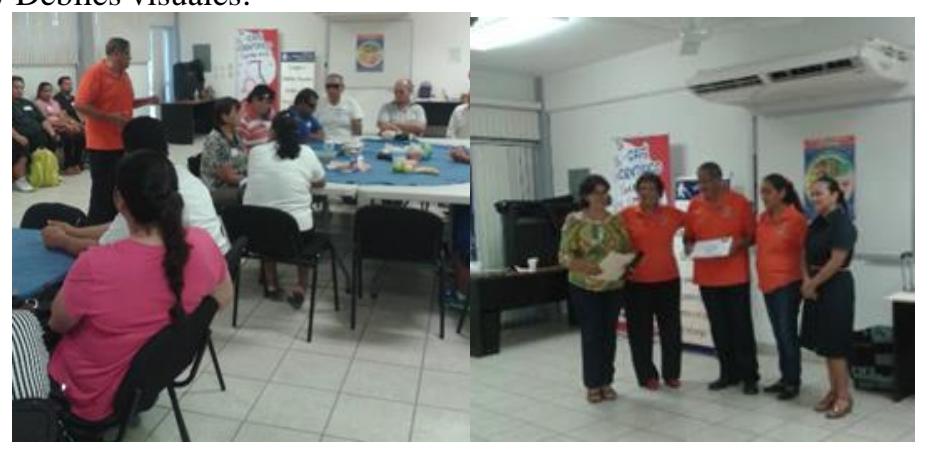

Para el Café Científico de octubre de 2015, se impartió el tema "Calentamiento Global o Glaciación” por el M. en C. Manuel Oseguera Cházaro Profesor/Investigador de la Universidad Autónoma de Baja California Sur. Una explicación del problema que a nivel global enfrentamos por el consumo excesivo de hidrocarburos y carbón que han desatado un cambio brusco en las temperaturas de la atmosfera y los océanos, modificando el clima mundial (Fig. 15). 
Figura 15.- En estos días nos encontramos en la nueva Era el "Antropoceno", cuyo impacto del humano sobre la biosfera ha marcado un cambio global de magnitudes significantes; es el mensaje que nos quiere dar el M. en C. Manuel Oseguera, tomar conciencia.

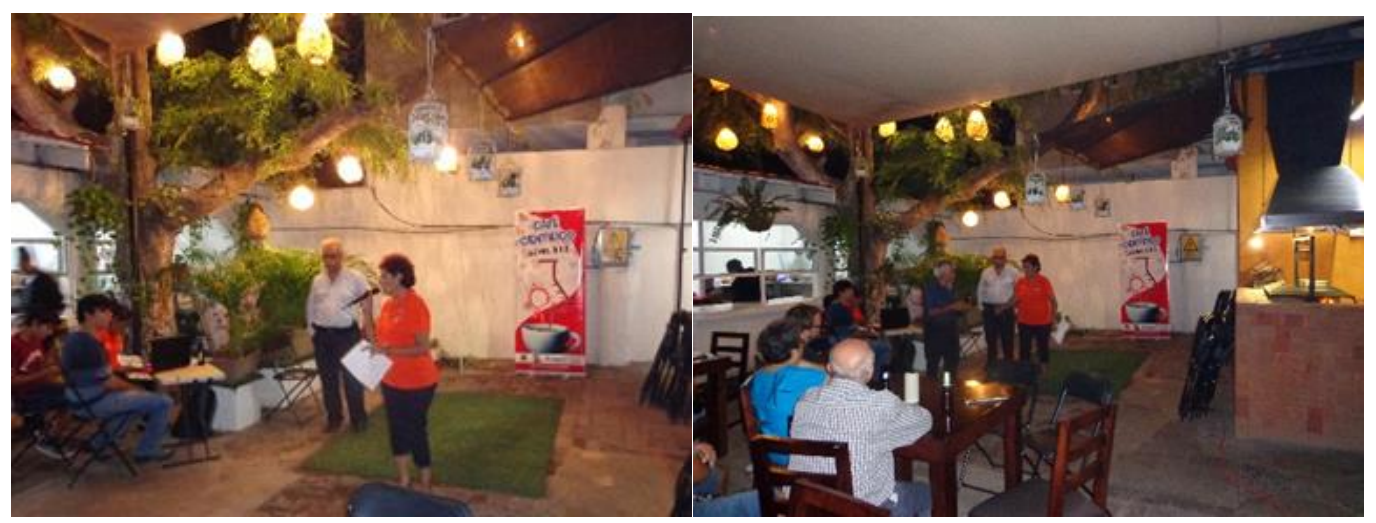

Nuestra última sesión del Café Científico fue en noviembre de 2015, con la charla de "Aprendo y Juego" impartida por el Geol. Alejandro Álvarez Arellano Profesor/Investigador de la Universidad Autónoma de Baja California Sur. El desarrollo de la plática fue con la ayuda de juguetes didácticos elaborados con madera por el mismo ponente (Fig. 16).

Figura 16.- Una charla que el motivo fue completamente lúdico, el ponente nos mostró unos juguetes didácticos que tienen el fin de entretenimiento para personas de distintas edades.

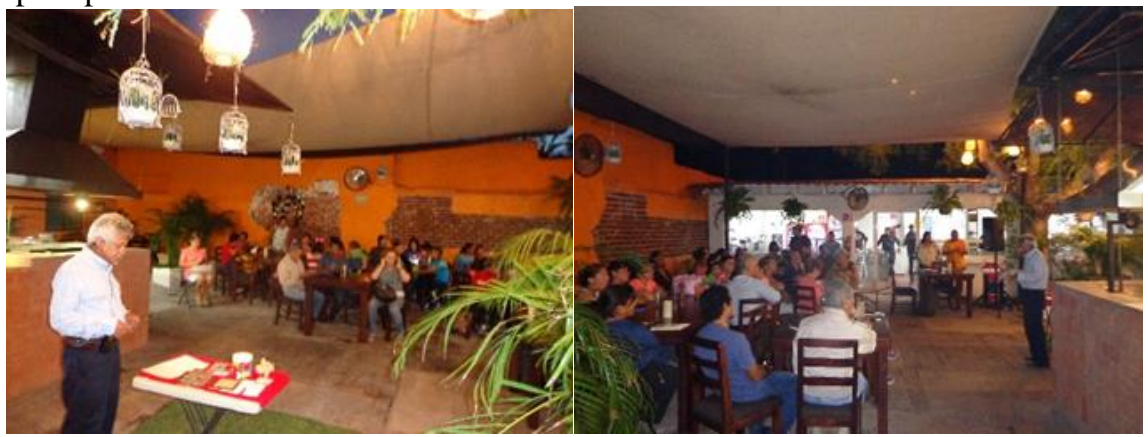

A través de YouTube; las sesiones del Café Científico quedan grabadas. Además, se invita a las sesiones y se tiene comunicación constante a través de Facebook del correo electrónico y redes sociales https://www.facebook.com/CafeCientificoLaPaz.

En la página internacional que reúne a los cafés científicos del mundo: http://www.cafecientifique.org/bajacalifornia ${ }_{2}$ para dar una difusión más amplia. 


\section{CONCLUSIONES}

En este ciclo anual, tuvimos trece sesiones de Café Científico de las cuales tres de ellas fueron sesiones por invitaciones especiales que se realizaron en diferentes salas de las organizaciones y las diez que tienen lugar en el restaurante sede de la ocasión.

El alcance de este periodo fue notable y fácil de identificar por: asistencia a las sesiones, importante mencionar que la sesión de marzo se difirió una semana por estar cerrado el local, no obstante, la cantidad de asistentes fue mayor que en otras sesiones.

Las sesiones con la modalidad del ¡Café Científico sale a la calle! en Telebachillerato 07 San Pedro y en otros sitios, fue una grata experiencia tanto para los ponentes como para los asistentes, permitió compartir conocimientos actuales de cómo mantener una alimentación saludable, con acciones prácticas y productos tangibles reales que los mismos asistentes aportaron. 


\section{REFERENCIAS BIBLIOGRÁFICAS}

Juárez Olvera, C. del C., Álvarez Ramírez, I. M., Félix Pico, E.F. y Piña Puente, J.F. (2015) Informe de actividades y financiero del Programa de Apoyo a las culturas Municipales y Comunitarias (PACMYC 2014), mediante el proyecto denominado Café Científico La Paz de CODICE A.C. p. 10.

Juárez Olvera, C. del C., Álvarez Ramírez, I. M., Piña Puente, J.F. y Félix Pico, E.F. (2016) Programa para las Culturas Populares Municipales, Secretaria de Cultura y el Café Científico La Paz. In: Resúmenes del XXI Congreso Nacional de Divulgación de la Ciencia y la Técnica. Comunicación Pública de la Ciencia en la Era Digital, SOMEDICyT, A.C. Del 30 de agosto al 2 de septiembre de 2016, Ciudad de Querétaro, México. P. 93-94.

Red internacional de Cafés Científicos, a través del sitio http://www.cafecientifique.org/bajacalifornia. Sociedad Mexicana para la Divulgación de la Ciencia y la Técnica, A.C. (SOMEDICYT A.C.), sitio https://somedicyt.org.mx/ . 